\title{
On the number of eigenvalues of the discrete one-dimensional Schrödinger operator with a complex potential
}

\author{
Artem Hulko $^{1}$ (D)
}

Received: 17 October 2016 / Accepted: 9 November 2016 / Published online: 21 November 2016

(C) The Author(s) 2016. This article is published with open access at Springerlink.com

\begin{abstract}
We study the eigenvalues of the discrete Schrödinger operator with a complex potential. We obtain bounds on the total number of eigenvalues in the case where $V$ decays exponentially at infinity.
\end{abstract}

Keywords Discrete Schrödinger · Complex potential · Total number of eigenvalues

\section{Introduction and main results}

Let $\mathfrak{H}=\ell^{2}\left(\mathbb{Z}_{+}\right)$be the Hilbert space of square summable sequences on $\mathbb{Z}_{+}=$ $\{1,2,3, \ldots\}$. Let $V: \mathfrak{H} \mapsto \mathfrak{H}$ be the operator of multiplication by a bounded complexvalued function on $\mathbb{Z}_{+}$. We study the spectral properties of the Schrödinger operator $H$, defined in $\mathfrak{H}$ by

$$
(H u)_{j}=\sum_{|l-j|=1} u_{l}+V_{j} u_{j}, \quad \forall j \geq 2 .
$$

Additionally, we set

$$
(H u)_{1}=u_{2}+V_{1} u_{1}
$$

Communicated by Ari Laptev.

$\bowtie$ Artem Hulko

ahulko@uncc.edu

1 Department of Mathematics and Statistics, University of North Carolina at Charlotte, Charlotte, NC 28223, USA 
Note that $H$ is a bounded operator. The spectrum of the self-adjoint operator $H_{0}=$ $H-V$ coincides with the interval $[-2,2]$ and is absolutely continuous. Let $\lambda_{j}$ denote the eigenvalues of the operator (1.1). We are interested in an estimate of the total number $N$ of eigenvalues $\lambda_{j}$ in the case where the sequence $V_{j}$ decays exponentially fast.

More precisely, we shall prove the following two theorems:

Theorem 1.1 The number $N$ of eigenvalues of $H$ in $\ell^{2}\left(\mathbb{Z}_{+}\right)$, counting algebraic multiplicities, satisfies

$$
N \leq \frac{1}{2 \ln \Lambda}\left(\frac{2 \Lambda^{2}}{\Lambda^{2}-1} \sum_{n=1}^{\infty} \Lambda^{2 n}\left|V_{n}\right|\right)^{2}
$$

for any $\Lambda>1$.

A similar result for a continuous operator was proved in [13] by Frank, Laptev and Safronov.

We also establish a slightly different estimate:

Theorem 1.2 The number $N$ of eigenvalues of $H$ in $\ell^{2}\left(\mathbb{Z}_{+}\right)$, counting algebraic multiplicities, satisfies

$$
N \leq \frac{1}{\ln \Lambda} \frac{\Lambda^{2}}{\left(\Lambda^{2}-1\right)}\left(\sum_{n=1}^{\infty} \Lambda^{n}\left|V_{n}\right|^{1 / 2}\right)^{2}
$$

for any $\Lambda>1$.

Note that the right hand sides of both estimates can be finite only in the case where $V$ is an exponentially decaying potential. It turns out that $N$ might be finite even in the case when the potential decays slower. For instance, the operator $-d^{2} / d x^{2}+V(x)$ on the half-line $[0, \infty)$ has finitely many eigenvalues if $|V| \leq C \exp (-c \sqrt{x})$ for some $C, c>0$. This remarkable result was proved by Pavlov in [23]. It was established that the eigenvalues can not accumulate to a point of the positive half- line, which is enough to conclute that the set of all eigenvalues is finite.

On the other hand, there is another remarkable result of Pavlov (see [24]), which says that, for any $0<p<1 / 2$, there exists a complex-valued potential $V$ satisfying $|V| \leq C \exp \left(-c|x|^{p}\right)$ and a complex number $\theta$, such that the operator $-d^{2} / d x^{2}+V(x)$ with the boundary condition $\psi^{\prime}(0)=\theta \psi(0)$ has infinitely many eigenvalues. Another interesting result was recently established by Bögli [2]. It was shown that there exists a potential for which the eigenvalues accumulate to every point on $[0, \infty)$.

\section{Zeroes of analytic functions}

The following proposition gives a useful bound on the zeroes of an analytic function in the compliment of the disc of radius $R>0$. 
Proposition 2.1 Let $0<R<1$. Let $a(\cdot)$ be an analytic function in $\{k:|k|>R\}$. Assume that $a(\cdot)$ is continuous up to the boundary and satisfies

$$
a(k)=1+O\left(|k|^{-1}\right) \text { as }|k| \rightarrow \infty \text { in }\{k:|k|>R\} .
$$

Assume also that for some $A \geq 1$,

$$
|a(k)| \leq A, \quad \text { if }|k|=R
$$

Then the zeroes $k_{j}$ of $a(\cdot)$ in $\{k:|k|>R\}$, repeated according to their multiplicities, satisfy

$$
\prod_{j}\left(\frac{\left|k_{j}\right|}{R}\right) \leq A
$$

Proof We introduce the Blaschke product

$$
B(k)=\prod_{j} \frac{k-k_{j}}{R-R^{-1} \overline{k_{j}} k} .
$$

Clearly, $a(k) / B(k)$ is an analytic and non-zero in $\{k:|k|>R\}$. Consequently, $\log (a(k) / B(k))$ exists and is analytic in $\{k:|k|>R\}$. Let $C_{R}$ denote the circle $\{k \in \mathbb{C}:|k|=R\}$, traversed counterclockwise.

Then, according to the residue calculus,

$$
\int_{C_{R}} \log \frac{a(k)}{B(k)} \frac{d k}{k}=2 \pi i \lim _{k \rightarrow \infty} \log \frac{a(k)}{B(k)}=2 \pi i \sum_{j} \log \frac{\bar{k}_{j}}{-R},
$$

and therefore

$$
\int_{-\pi}^{\pi} \log \frac{a\left(R e^{i \varphi}\right)}{B\left(R e^{i \varphi}\right)} d \varphi=2 \pi \sum_{j} \log \frac{\bar{k}_{j}}{-R} .
$$

We note that $\left|B\left(R e^{i \varphi}\right)\right|=1$ if $\varphi \in \mathbb{R}$ and, therefore,

$$
\operatorname{Re} \int_{-\pi}^{\pi} \log \frac{a\left(R e^{i \varphi}\right)}{B\left(R e^{i \varphi}\right)} d \varphi=\int_{-\pi}^{\pi} \ln \left|\frac{a\left(R e^{i \varphi}\right)}{B\left(R e^{i \varphi}\right)}\right| d \varphi=\int_{-\pi}^{\pi} \ln \left|a\left(R e^{i \varphi}\right)\right| d \varphi .
$$

On the other hand,

$$
\operatorname{Re} \sum_{j} \log \frac{\bar{k}_{j}}{-R}=\sum_{j} \ln \frac{\left|k_{j}\right|}{R} .
$$

We conclude from (2.4), (2.5) and (2.6) that

$$
\int_{-\pi}^{\pi} \ln \left|a\left(R e^{i \varphi}\right)\right| d \varphi=2 \pi \sum_{j} \ln \frac{\left|k_{j}\right|}{R} .
$$


Finally, by (2.2),

$$
\int_{-\pi}^{\pi} \ln \left|a\left(R e^{i \varphi}\right)\right| d \varphi \leq 2 \pi \ln A, .
$$

Inequality (2.3) now follows from (2.7) and (2.8).

Corollary 2.2 Let $0<R<1$. Let $a(\cdot)$ be an analytic function in $\{k:|k|>R\}$ satisfying (2.1). Assume that, for any $R^{\prime}>R$ sufficiently close to $R$, condition (2.2) holds with $R$ replaced by $R^{\prime}$. Then the number

$$
\mathcal{N}:=\#\left\{j:\left|k_{j}\right| \geq 1\right\}
$$

of zeroes $k_{j}$ of a $(\cdot)$ in $\{k:|k| \geq 1\}$, repeated according to their multiplicities, satisfies

$$
\mathcal{N} \leq \frac{\ln A}{\ln 1 / R}
$$

Proof We apply Proposition 2.1 for every $R^{\prime}>R$ sufficiently close to $R$ and obtain

$$
\sum_{j}\left(\ln \left|k_{j}\right|-\ln R^{\prime}\right)_{+} \leq \ln A
$$

Clearly, we have

$$
\sum_{j}\left(\ln \left|k_{j}\right|-\ln R^{\prime}\right)_{+} \geq\left|\ln R^{\prime}\right| \cdot \#\left\{j:\left|k_{j}\right| \geq 1\right\} .
$$

Consequently,

$$
\left|\ln R^{\prime}\right| \cdot \mathcal{N} \leq \ln A
$$

The corollary follows by passing to the limit $R^{\prime} \rightarrow R$.

\section{Classes of compact operators and determinants}

Let $1 \leq p<\infty$. We say that a compact operator $T$ belongs to the Schatten class $\mathfrak{S}_{p}$ if its singular values $s_{j}(T)$ satisfy

$$
\|T\|_{\mathfrak{S}_{p}}^{p}:=\sum_{j} s_{j}^{p}(T)<\infty .
$$

The functional $\|\cdot\|_{\mathfrak{S}_{p}}$ is the norm on $\mathfrak{S}_{p}$. 
Let $K \in \mathfrak{S}_{n}$ with $n \in \mathbb{N}$. Let $\lambda_{j}(K)$ denote the eigenvalues of $K$, repeated according

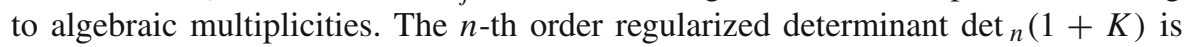
defined by

$$
\operatorname{det}_{n}(1+K):=\prod_{j}\left(\left(1+\lambda_{j}(K)\right) \exp \left(\sum_{m=1}^{n-1} \frac{(-1)^{m}}{m} \lambda_{j}(K)^{m}\right)\right) .
$$

The following property is well-known, but we include a proof for the sake of completeness.

Lemma 3.1 Let $n \in \mathbb{N}$ and let $K \in \mathfrak{S}_{n}$. Then

$$
\ln \left|\operatorname{det}_{n}(1+K)\right| \leq \Gamma_{n}\|K\|_{\mathfrak{S}_{n}}^{n},
$$

where $\Gamma_{n}$ is a positive constant independent of $K$. In particular,

$$
\Gamma_{1}=1 \text { and } \Gamma_{2}=1 / 2 \text {. }
$$

Proof To prove the lemma, let $f(z):=(1+z) \exp \left(\sum_{m=1}^{n-1} \frac{(-1)^{m}}{m} z^{m}\right)$. Then $\ln |f(z)|$ can be bounded by a constant times $|z|^{n}$ for small $|z|$ and by a constant times $|z|^{n-1}$ for large $|z|$. Thus, $\ln |f(z)| \leq \Gamma_{n}|z|^{n}$, and so

$$
\ln \left|\operatorname{det}_{n}(1+K)\right| \leq \Gamma_{n} \sum_{j}\left|\lambda_{j}(K)\right|^{n}
$$

By Weyl's inequality [26, Thm. 1.15], the sum on the right side does not exceed $\|K\|_{\mathfrak{S}_{n}}^{n}$. A simple computation shows that for $n=1$ and $n=2$ one can take $\Gamma_{1}=1$ and $\Gamma_{2}=1 / 2$, respectively (see [27]).

We now recall the Birman-Schwinger principle. We state it in the case, where $H_{0}$ is a bounded self-adjoint operator and $V=G^{*} G_{0}$. We will assume that $G_{0}$ and $G$ are compact operators. Now, set

$$
H=H_{0}+V \text {. }
$$

The Birman-Schwinger principle states that $z \in \rho\left(H_{0}\right)$ is an eigenvalue of $H$ if and only if -1 is an eigenvalue of the Birman-Schwinger operator $G_{0}\left(H_{0}-z\right)^{-1} G^{*}$. Moreover, the corresponding geometric multiplicities coincide.

The following lemma says that even the algebraic multiplicities of eigenvalues of $H$ can be characterizes in terms of a quantity related to the Birman-Schwinger operator.

Lemma 3.2 Let $n \in \mathbb{N}$. Assume that $G_{0}\left(H_{0}-\zeta\right)^{-1} G^{*} \in \mathfrak{S}_{n}$ for all $\zeta \in \rho\left(H_{0}\right)$. Then the function $\zeta \mapsto \operatorname{det}_{n}\left(1+G_{0}\left(H_{0}-\zeta\right)^{-1} G^{*}\right)$ is analytic in $\rho\left(H_{0}\right)$. A point $z \in \rho\left(H_{0}\right)$ is an eigenvalue of $H$ if and only if $\operatorname{det}_{n}\left(1+G_{0}\left(H_{0}-z\right)^{-1} G^{*}\right)=0$. Moreover, the order of the zero coincides with the algebraic multiplicity of the corresponding eigenvalue. 
Analyticity of the function $\zeta \mapsto \operatorname{det}_{n}\left(1+G_{0}\left(H_{0}-\zeta\right)^{-1} G^{*}\right)$ is well-known (see, e.g., [27]), as well as the result about the algebraic multiplicity in the case $n=1$. The result for the general $n$ is essentially due to [18]; you may also refer to [11] for an extension of the proof to the present setting.

\section{Resolvent bounds}

In this section we collect trace ideal bounds for the Birman-Schwinger operator

$$
K(k)=\sqrt{V}\left(H_{0}-z\right)^{-1} \sqrt{|V|}, \quad z=k+k^{-1}, \quad|k| \geq 1 .
$$

We use the notation $\sqrt{V(x)}=V(x) / \sqrt{|V(x)|}$ if $V(x) \neq 0$ and $\sqrt{V(x)}=0$ if $V(x)=0$.

We remind the reader that $\mathfrak{H}=\ell^{2}\left(\mathbb{Z}_{+}\right)$, and $H_{0}$ in (4.1) denotes the free Jacobi operator on $\mathbb{Z}_{+}$. From the explicit expression of its matrix it is easy to see that, if $V$ has a compact support, then $K(k)$ admits an analytic continuation to $\mathbb{C} \backslash\{0\}$. The following proposition gives a bound on the Hilbert-Schmidt norm.

Proposition 4.1 For any $k \in \mathbb{C} \backslash\{0\}$ with $|k|<1$,

$$
\|K(k)\|_{\mathfrak{S}_{2}} \leq \frac{2}{1-|k|^{2}} \sum_{n=1}^{\infty}|k|^{-2 n}\left|V_{n}\right|,
$$

Proof The matrix of $\left(H_{0}-z\right)^{-1}$ is given by

$$
g_{k}(n, m)=\frac{k}{k^{2}-1}\left(k^{-|n-m|}-k^{-(n+m)}\right),
$$

which satisfies

$$
\left|g_{k}(n, m)\right| \leq \frac{2}{1-|k|^{2}}|k|^{-(n+m)} .
$$

Combining this bound with the identity

$$
\|K(k)\|_{\mathfrak{S}_{2}}^{2}=\sum_{1}^{\infty} \sum_{1}^{\infty}\left|V_{n}\right|\left|g_{k}(n, m)\right|^{2}\left|V_{m}\right|
$$

we obtain the claimed bound.

Proposition 4.2 For any $k \in \mathbb{C} \backslash\{0\}$ with $|k|<1$,

$$
\|K(k)\|_{\mathfrak{S}_{1}} \leq \frac{2}{1-|k|^{2}}\left(\sum_{n=1}^{\infty}|k|^{-n}\left|V_{n}\right|^{1 / 2}\right)^{2}
$$


Proof The matrix of $\left(H_{0}-z\right)^{-1}$ is defined by

$$
g_{k}(n, m)=\frac{k}{k^{2}-1}\left(k^{-|n-m|}-k^{-(n+m)}\right),
$$

which satisfies

$$
\left|g_{k}(n, m)\right| \leq \frac{2}{1-|k|^{2}}|k|^{-(n+m)} .
$$

Combining this bound with the identity

$$
\|K(k)\| \mathfrak{S}_{1} \leq\left.\sum_{1}^{\infty} \sum_{1}^{\infty}\left|V_{n}\right|^{1 / 2}\left|g_{k}(n, m) \|\right| V_{m}\right|^{1 / 2}
$$

we obtain the claimed bound.

\section{Proof of Theorem 1.1}

In this section we prove Theorem 1.1. Let us assume that $V$ has compact support. The bound in this case implies the bound in the general case by a simple continuity argument.

As discussed in Sect. 4, the Birman-Schwinger operators $K(k)$ from (4.1) extends analytically to $\mathbb{C} \backslash\{0\}$. The same proof shows that they are not only analytic with respect to the norm of bounded operators, but even with respect to the norm in $\mathfrak{S}_{2}$.

We will apply Corollary 2.2 to the function

$$
a(k):=\operatorname{det}_{2}(1+K(k))
$$

with $\Lambda=1 / R$. Since $K(k)$ is analytic with values in $\mathfrak{S}_{2}$, the function $a$ is analytic. It is easy to see that assumption (2.1) is valid. Moreover, combining them with Lemma 3.1 , we see that assumption (2.2) holds with

$$
\ln A=\frac{1}{2}\left(\frac{2 \Lambda^{2}}{\Lambda^{2}-1} \sum_{n=1}^{\infty} \Lambda^{2 n}\left|V_{n}\right|\right)^{2}
$$

Thus, Corollary 2.2 implies that

$$
\#\left\{j: \quad \operatorname{Im} k_{j} \geq 0\right\} \leq \frac{1}{2 \ln \Lambda}\left(\frac{2 \Lambda^{2}}{\Lambda^{2}-1} \sum_{n=1}^{\infty} \Lambda^{2 n}\left|V_{n}\right|\right)^{2} .
$$

It remains to use Lemma 3.2, which says that the $k_{j}+k_{j}^{-1}$, with $\left|k_{j}\right|>1$, coincide with the eigenvalues of $H$, counting algebraic multiplicities. This proves Theorem 1.1. 


\section{Proof of Theorem 1.2}

In this section we prove Theorem 1.2. Let us assume again that $V$ has compact support.

As discussed in Sect. 4, the Birman-Schwinger operators $K(k)$ from (4.1) extend analytically to $\mathbb{C} \backslash\{0\}$. The same proof shows that they are not only analytic with respect to the norm of bounded operators, but even with respect to the norm in $\mathfrak{S}_{1}$.

We apply Corollary 2.2 to the function

$$
a(k):=\operatorname{det}_{1}(1+K(k))=\operatorname{det}(1+K(k))
$$

with $\Lambda=1 / R$. Since $K(k)$ is analytic with values in $\mathfrak{S}_{1}$, the function $a$ is analytic. Assumption (2.2) holds with

$$
\ln A=\left(\frac{2 \Lambda^{2}}{\Lambda^{2}-1} \sum_{n=1}^{\infty} \Lambda^{n}\left|V_{n}\right|^{1 / 2}\right)^{2}
$$

Thus, Corollary 2.2 implies that

$$
\#\left\{j: \quad \operatorname{Im} k_{j} \geq 0\right\} \leq \frac{1}{\ln \Lambda}\left(\frac{2 \Lambda^{2}}{\Lambda^{2}-1} \sum_{n=1}^{\infty} \Lambda^{n}\left|V_{n}\right|^{1 / 2}\right)^{2}
$$

It remains to use Lemma 3.2, which says that the $k_{j}+k_{j}^{-1}$, with $\left|k_{j}\right|>1$, coincide with the eigenvalues of $H$, counting algebraic multiplicities. This proves Theorem 1.2.

Most of the papers listed below contain results on the eigenvalues of non-selfadjoint operators. More specifically, those are the articles [1-17, 19-25, 28, 29]. The remaining references were needed for technical reasons.

Open Access This article is distributed under the terms of the Creative Commons Attribution 4.0 International License (http://creativecommons.org/licenses/by/4.0/), which permits unrestricted use, distribution, and reproduction in any medium, provided you give appropriate credit to the original author(s) and the source, provide a link to the Creative Commons license, and indicate if changes were made.

\section{References}

1. Abramov, A.A., Aslanyan, A., Davies, E.B.: Bounds on complex eigenvalues and resonances. J. Phys. A 34, 57-72 (2001)

2. Bögli, S.: Schrödinger operator with non-zero accumulation points of complex Eigenvalues (2016). arxiv:1605.09356 (Preprint)

3. Borichev, A., Golinskii, L., Kupin, S.: A Blaschke-type condition and its application to complex Jacobi matrices. Bull. Lond. Math. Soc. 41, 117-123 (2009)

4. Davies, E.B.: Non-self-adjoint differential operators. Bull. Lond. Math. Soc. 34(5), 513-532 (2002)

5. Davies, E.B., Nath, J.: Schrödinger operators with slowly decaying potentials. J. Comput. Appl. Math. 148, 1-28 (2002)

6. Demuth, M., Katriel, G.: Eigenvalue inequalities in terms of Schatten norm bounds on differences of semigroups, and application to Schrödinger operators. Ann. Henri Poincaré 9(4), 817-834 (2008) 
7. Demuth, M., Hansmann, M., Katriel, G.: On the discrete spectrum of non-selfadjoint operators. J. Funct. Anal. 257(9), 2742-2759 (2009)

8. Demuth, M., Hansmann, M., Katriel, G.: Eigen values of non-self adjoint operators: a comparison of two approaches. In: Mathematical Physics, Spectral Theory and Stochastic Analysis, Springer, pp. 107-163 (2013)

9. Enblom, A.: Estimates for eigenvalues of Schrödinger operators with complex-valued potentials (2015). arxiv:1503.06337 (Preprint)

10. Frank, R.L.: Eigenvalue bounds for Schrödinger operators with complex potentials. Bull. Lond. Math. Soc. 43(4), 745-750 (2011)

11. Frank, R.L.: Eigenvalue bounds for Schrödinger operators with complex potentials. III (2015). arxiv:1510.03411v1 (Preprint)

12. Frank, R.L., Laptev, A., Lieb, E.H., Seiringer, R.: Lieb-Thirring inequalities for Schrödinger operators with complex-valued potentials. Lett. Math. Phys. 77, 309-316 (2006)

13. Frank, R.L., Laptev, A., Safronov, O.: On the number of eigenvalues of Schrödinger operators with complex potentials. to appear

14. Frank, R.L., Laptev, A., Seiringer, R.: A sharp bound on eigenvalues of Schrödinger operators on the half-line with complex-valued potentials. Spectral theory and analysis, pp. 39-44, Oper. Theory Adv. Appl. 214, Birkhäuser/Springer Basel AG, Basel (2011)

15. Frank, R.L., Sabin, J.: Restriction theorems for orthonormal functions, Strichartz inequalities and uniform Sobolev estimates (2014). arxiv:1404.2817 (Preprint)

16. Frank, R.L., Simon, B.: Eigenvalue bounds for Schrödinger operators with complex potentials. II. J. Spectr. Theory (to appear)

17. Laptev, A., Safronov, O.: Eigenvalue estimates for Schrödinger operators with complex potentials. Comm. Math. Phys. 292, 29-54 (2009)

18. Latushkin, Y., Sukhtayev, A.: The algebraic multiplicity of eigenvalues and the Evans function revisited. Math. Model. Nat. Phenom. 5(4), 269-292 (2010)

19. Martirosjan, R.M.: On the spectrum of the non-selfadjoint operator $\Delta u+c u$ in three dimensional space. (Russian) Izv. Akad. Nauk Armyan. SSR. Ser. Fiz.-Mat. Nauk 10(1), 85-111 (1957)

20. Martirosjan, R.M.: On the spectrum of various perturbations of the Laplace operator in spaces of three or more dimensions. (Russian) Izv. Akad. Nauk SSSR Ser. Mat. 24, 897-920 (1960)

21. Murtazin, K.K.: Spectrum of the nonself-adjoint Schrödinger operator in unbounded regions. (Russian) Mat. Zametki 9, 19-26. English translation: Math. Notes 9(1971), 12-16 (1971)

22. Nămark, M.A.: Investigation of the spectrum and the expansion in eigenfunctions of a nonselfadjoint operator of the second order on a semi-axis. (Russian) Trudy Moskov. Mat. Obšč. 3, 181-270 (1954)

23. Pavlov, B.S.: On a non-selfadjoint Schrödinger operator. (Russian) 1966 Probl. Math. Phys., No. 1, Spectral Theory and Wave Processes (Russian) pp. 102-132 Izdat. Leningrad. Univ., Leningrad

24. Pavlov, B.S.: On a non-selfadjoint Schrödinger operator. II. (Russian) 1967 Problems of Mathematical Physics, No. 2, Spectral Theory, Diffraction Problems (Russian) pp. 133-157 Izdat. Leningrad. Univ., Leningrad

25. Safronov, O.: On a sum rule for Schrödinger operators with complex potentials. Proc. Am. Math. Soc. 138(6), 2107-2112 (2010)

26. Simon, B.: Trace Ideals and Their Applications, 2nd edn. Amer. Math. Soc, Providence (2005)

27. Simon, B.: Notes on infinite determinants of Hilbert space operators. Adv. Math. 24(3), 244-273 (1977)

28. Stepin, S.A.: Complex potentials: bound states, quantum dynamics and wave operators. Semigroups of operators - theory and applications, 287-297, Springer Proc. Math. Stat. 113, Springer, Cham (2015)

29. Stepin, S.A.: Estimate for the number of eigenvalues of the nonselfadjoint Schrödinger operator. (Russian) Dokl. Akad. Nauk 455(4), 394-397; translation in Dokl. Math. 89(2), 202-205 (2014) 